\title{
Channel Modeling for IRS-Assisted FSO
}

\section{Systems}

\author{
Hedieh Ajam, Marzieh Najafi, Vahid Jamali, and Robert Schober \\ Friedrich-Alexander University Erlangen-Nuremberg, Germany
}

\begin{abstract}
In this paper, we develop an analytical channel model for intelligent reflecting surface (IRS)-assisted free space optical (FSO) systems. Unlike IRS-assisted radio frequency systems, where it is typically assumed that a plane wave is incident on the IRS, in FSO systems, the incident wave is a Gaussian beam with non-uniform power distribution across the IRS. Taking this property into account, we develop an analytical end-to-end channel model for IRS-assisted FSO systems based on the Huygens-Fresnel principle. Our analytical model reveals the impact of the size, position, orientation, and phase-shift configuration of the IRS on the end-to-end channel. Furthermore, we show that results obtained based on geometric optics under the far-field approximation are only valid for a specific range of IRS-receiver lens distances depending on the IRS size, incident beam width, and wavelength. Simulation results validate the accuracy of the proposed analytical results for the FSO beam reflected from the IRS and compare the bit error rate performance obtained for the proposed analytical channel model with that obtained for geometric optics under the far-field approximation.
\end{abstract}

\section{INTRODUCTION}

Metamaterials can manipulate the properties of a wave such as its polarization, phase, and amplitude in reflection and transmission [1], [2]. Intelligent reflecting surfaces (IRSs) are planar structures of metamaterials with subwavelength thickness and consist of many subwavelength elements referred to as unit cells [1]. In order to achieve a desired behavior, the IRS changes the accumulated phase of the wave reflected by the surface. The phase shifts applied by the unit cells determine the characteristics of the IRS. In the literature, typically a linear phase shift gradient is assumed, so that the IRS can provide anomalous reflection of a beam in a desired direction [3]-[5]. In radio frequency (RF) wireless communication systems, IRS have been exploited to increase coverage, ensure security, harness interference, and improve the quality of non-line-ofsight (NLOS) connections [6]. For free space optical (FSO) systems, which require in general 
a line-of-sight (LOS) connection, the authors of [7] employed an optical IRS to connect a transmitter with an obstructed receiver. Unlike RF waves, which have a plane wavefront and uniform power distribution across the IRS, the Gaussian beams employed in FSO systems have a curved wavefront and a non-uniform power distribution. The authors of [7] exploited geometric optics using a far-field approximation to determine the impact of IRSs on the performance of FSO systems. In this paper, we will show that this approach is only valid for specific IRS-receiver lens distances, incident beam widths, and IRS sizes. Moreover, the authors of [8] applied an optical IRS to enhance an indoor communication link. In [9], the impact of IRSs on visible light communications (VLC) was investigated. However, VLC employs non-directional beams which exhibit a different behavior compared to the Gaussian laser beams used in FSO systems.

In this paper, we employ an IRS-assisted FSO system to provide an LOS connection between a transmitter and an obstructed receiver. The transmitter is equipped with a laser source (LS) emitting a Gaussian beam which is reflected by an IRS towards the receiver where the beam is focused by a lens onto a photo detector (PD). Based on the Huygens-Fresnel principle, we analyze the channel gain of this IRS-assisted FSO system taking the non-uniform power distribution of the Gaussian beam into account. In the following, we summarize the main contributions of our work.

- Based on the Huygens-Fresnel principle, we derive the deterministic channel gain of an IRS-assisted FSO system which employs a Gaussian beam that is emitted by an LS and is reflected by an optical IRS. The impact of the relative position of the IRS with respect to (w.r.t.) the lens and the LS, the size of the IRS, and the phase-shift configuration of the IRS are included in the proposed analytical end-to-end channel model.

- In the far-field approximation, the wave reflected by the IRS is modeled as parallel rays with linear phase differences. However, we will show that depending on the IRS size and the incident beam width, this approximation may not always be valid. We mathematically characterize the range of intermediate and far-field distances and propose an analytical channel model that is valid for these distances. We show that large distances (e.g. more than $32 \mathrm{~km}$ for a square-shaped IRS of length $0.5 \mathrm{~m}$ and incident beam widths of $0.19 \mathrm{~m}$ and $0.52 \mathrm{~m}$ ) may be needed for the far-field approximation to be valid.

- Our simulation results validate the analytical results for the electric field of the beam reflected by the IRS and the deterministic gain of the IRS-assisted FSO channel. Moreover, we compare the bit error rate (BER) performance of an IRS-assisted FSO system for the 


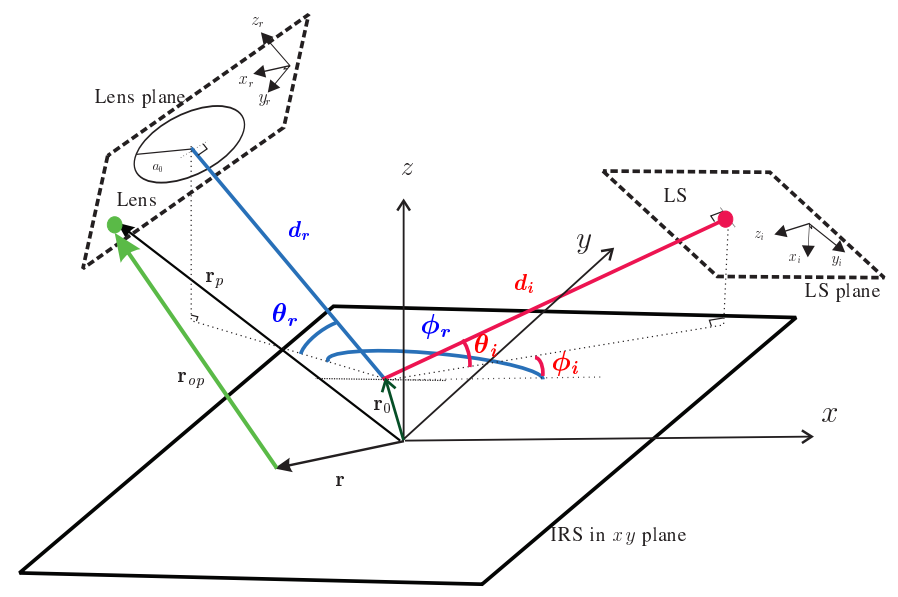

Fig. 1. Schematic model of IRS-assisted FSO system.

developed analytical channel model with results obtained for the channel model based on geometric optics under the far-field approximation. Our simulation results confirm that for larger IRS sizes and beam widths and practical distances, the far-field approximation is not valid and the proposed model has to be used.

Notations: Boldface lower-case and upper-case letters denote vectors and matrices, respectively. Superscript $(\cdot)^{T}$ and $\mathbb{E}\{\cdot\}$ denote the transpose and expectation operators, respectively. $\mathbf{I}_{n}$ is the $n \times n$ identity matrix, $j$ denotes the imaginary unit, and $\operatorname{conj}(\cdot)$ and real $(\cdot)$ represent the complex conjugate and the real part of a complex number, respectively. Moreover, $\operatorname{erf}(\cdot)$ and $\operatorname{erfi}(\cdot)$ are the error function and the imaginary error function, respectively.

\section{System Model}

We consider an IRS-assisted FSO system where a transmitter is equipped with an LS to communicate through an IRS with a receiver equipped with a PD and a lens, see Fig. 1. In particular, the LS illuminates the IRS with a Gaussian laser beam and the receiver collects the power reflected by the IRS with its lens and detects the received power with its PD [7]. The IRS is positioned in the $x y$-plane and centered at the origin. It has length $L_{x}$ in $x$-direction and width $L_{y}$ in $y$-direction. The IRS consists of many subwavelength unit cells and by properly adjusting their phases, the IRS is able to reflect the incident beam from the LS towards the receiver. Given that the IRS is larger than the beam wavelength, i.e., $L_{x}, L_{y} \gg \lambda$, it can be modeled as a continuous surface with continuous phase-shift configuration, denoted by $\Phi_{\text {irs }}[3]$. Here, we assume a linear phase gradient across the unit cells, similar to previous related works [3], [10], 
see Section III for more details. In the considered system, the LS is located at $\mathbf{p}_{i}=\left(d_{i}, \theta_{i}, \phi_{i}\right)$, where $d_{i}$ is the distance between the LS and the beam footprint center on the IRS along the beam axis, $\theta_{i}$ is the angle between the $x y$-plane and the beam axis, and $\phi_{i}$ is the angle between the projection of the beam axis on the $x y$-plane and the $x$-axis. Without loss of generality, we assume $\phi_{i}=0$ in the following. Moreover, the beam footprint on the IRS plane is centered at point $\mathbf{r}_{0}=\left(x_{0}, y_{0}, 0\right)$. Furthermore, we assume the receiver is equipped with a circular lens of radius $a_{0}$ which is always perpendicular to the received beam axis and the lens center is positioned at $\mathbf{p}_{r}=\left(d_{r}, \theta_{r}, \phi_{r}\right)$, where $d_{r}$ is the distance between the beam footprint center on the IRS and the lens center, $\theta_{r}$ is the angle between the $x y$-plane and the normal vector of the lens plane, and $\phi_{r}$ is the angle between the projection of the normal vector of the lens plane on the $x y$-plane and the $x$-axis.

At the receiver, the lens focuses the beam, which was reflected by the IRS, onto the PD and the signal $y$ received at the PD can be modeled as

$$
y=h s+n,
$$

where $s$ is an on-off keying (OOK) modulated optical symbol with average power $\sigma_{s}^{2}=\mathbb{E}\left\{|s|^{2}\right\}$, $h \in \mathcal{R}^{+}$is the channel gain, and $n$ is additive white Gaussian noise (AWGN) with zero mean and variance $\sigma_{n}^{2}$. In general, FSO channels are affected by geometric and misalignment losses, atmospheric losses, and atmospheric turbulence [11]. For simplicity, we assume perfect beam tracking and that the positions of the IRS, LS, and lens do not fluctuate. Thus, the misalignment loss can be ignored, see [7] for an analysis of the misalignment loss of IRS-assisted FSO systems. Then, the IRS-assisted FSO channel gain $h$ between the LS and the PD can be modeled as

$$
h=h_{p} h_{\mathrm{irs}} h_{a}
$$

where $h_{a}$ is the random atmospheric turbulence component, $h_{p}=10^{-\frac{\kappa}{10}\left(d_{i}+d_{r}\right)}$ is the atmospheric loss dependent on attenuation factor, $\kappa$, and $h_{\text {irs }}$ is the geometric loss. We assume that $h_{a}$ is Gamma-Gamma distributed, i.e., $h_{a} \sim \mathcal{G G}(\alpha, \beta)$, where $\alpha$ and $\beta$ are the small and large scale turbulence parameters [12]. Moreover, $h_{\mathrm{irs}}$ denotes the fraction of power reflected by the IRS and collected by the PD and is given by

$$
h_{\mathrm{irs}}=\iint_{\left(x_{r}, y_{r}\right) \in \mathcal{A}} I_{\mathrm{irs}}\left(\mathbf{r}_{r}\right) \mathrm{d} x_{r} \mathrm{~d} y_{r},
$$


where $\mathcal{A}$ is the area of the lens and $\mathbf{r}_{r}=\left(x_{r}, y_{r}, z_{r}\right)$ denotes the coordinates on the lens plane. The origin of the $x_{r} y_{r} z_{r}$-coordinate system is located at the center of the lens and the $z_{r}$-axis points in the opposite direction of the normal vector of the lens plane, see Fig. 1. We assume that the $y_{r}$-axis is along the intersection line of the lens plane and the IRS plane and the $x_{r}$-axis is perpendicular to the $y_{r}$-axis. Here, $I_{\text {irs }}\left(\mathbf{r}_{r}\right)$ is the power density of the reflected beam in the lens plane. Given that the Gaussian laser beam waist, $w_{0}$, is larger than the wavelength, $\lambda$, the paraxial approximation is valid and the power density of the reflected beam can be expressed as follows [13]

$$
I_{\mathrm{irs}}\left(\mathbf{r}_{r}\right)=\frac{1}{2 \eta}\left|E_{r}\left(\mathbf{r}_{r}\right)\right|^{2}
$$

where $\eta$ is the free-space impedance and $E_{r}\left(\mathbf{r}_{r}\right)$ is the electric field reflected by the IRS and observed at the lens.

The electric field of the Gaussian laser beam emitted by the LS is given by [14]

$$
\begin{gathered}
E_{o}\left(\mathbf{r}_{i}\right)=\frac{E_{0} w_{0}}{w\left(z_{i}\right)} \exp \left(-\frac{x_{i}^{2}+y_{i}^{2}}{w^{2}\left(z_{i}\right)}-j \phi_{o}\right) \quad \text { with phase } \\
\phi_{o}=k\left(z_{i}+\frac{x_{i}^{2}+y_{i}^{2}}{2 R\left(z_{i}\right)}\right)-\tan ^{-1}\left(\frac{z_{i}}{z_{0}}\right)
\end{gathered}
$$

where $\mathbf{r}_{i}=\left(x_{i}, y_{i}, z_{i}\right)$ is a point in a coordinate system which has its origin at the LS, the $z_{i}$-axis is along the beam axis, the $y_{i}$-axis is along the intersection line of the LS plane and the IRS plane, and the $x_{i}$-axis is orthogonal to the $y_{i}$-axis. Here, $E_{0}$ is the electric field at the origin, $k=\frac{2 \pi}{\lambda}$ is the wave number, $w\left(z_{i}\right)=w_{0}\left[1+\left(\frac{z_{i}}{z_{0}}\right)^{2}\right]^{1 / 2}$ is the beam width at distance $z_{i}, R\left(z_{i}\right)=z_{i}\left[1+\left(\frac{z_{0}}{z_{i}}\right)^{2}\right]$ is the radius of curvature of the beam's wavefront, and $z_{0}=\frac{\pi w_{0}^{2}}{\lambda}$ is the Rayleigh range.

\section{Proposed Channel Model}

Here, given the Gaussian laser beam in (5), first we determine the electric field incident on the IRS, then, we determine the reflected electric field $E_{r}\left(\mathbf{r}_{r}\right)$ using the Huygens-Fresnel principle. Next, we derive $I_{\text {irs }}\left(\mathbf{r}_{r}\right)$ and the corresponding channel gain $h_{\text {irs. }}$.

\section{A. Reflected Electric Field}

First, in the following lemma, we determine the incident electric field on the IRS plane. 
Lemma 1: Assuming that $d_{i} \gg L_{x}, L_{y}$, the electric field emitted by the LS incident on the IRS plane, denoted by $E_{i}(\mathbf{r})$, is given by

$$
\begin{aligned}
E_{i}(\mathbf{r}) & =\frac{E_{0} w_{0}}{w\left(\tilde{d}_{i}\right)} \exp \left(-\frac{\hat{x}^{2}+\hat{y}^{2}}{w^{2}\left(\tilde{d}_{i}\right)}-j \phi_{i n}\right) \quad \text { with phase } \\
\phi_{i n} & =k\left(\hat{d}_{i}+\frac{\hat{x}^{2}+\hat{y}^{2}}{2 R\left(\tilde{d}_{i}\right)}\right)-\tan ^{-1}\left(\frac{\tilde{d}_{i}}{z_{0}}\right),
\end{aligned}
$$

where $\mathbf{r}=(x, y, 0)$ denotes any point in the $x y$-plane, $\hat{d}_{i}=d_{i}+\left(x-x_{0}\right) \cos \left(\theta_{i}\right), \tilde{d}_{i}=d_{i}-$ $x_{0} \cos \left(\theta_{i}\right), \hat{x}=\sin \left(\theta_{i}\right)\left(x-x_{0}\right)$, and $\hat{y}=y-y_{0}$.

Proof: The proof is given in Appendix A

Eq. 6) determines an elliptical Gaussian beam on the IRS with beam widths $w_{x}=\frac{w\left(\tilde{d}_{i}\right)}{\sin \left(\theta_{i}\right)}$ and $w_{y}=w\left(\tilde{d}_{i}\right)$ along $x$ - and $y$-axes.

Next, to determine the impact of the IRS on the incident beam, we use scalar field theory [14] and neglect the vectorial nature of the electromagnetic field. This approach yields accurate result if the following conditions are met: 1) the diffracting surface must be large compared to the wavelength, 2) the electromagnetic fields must not be observed very close to the surface, i.e., $d_{r} \gg \lambda[14]$. Given the size of the IRS and the application of FSO systems for long-distance communications, these conditions are met in practice. Thus, we can apply the Huygens-Fresnel principle for deriving the beam reflected by the IRS. This principle states that every point on the wavefront of the beam can be considered as a secondary source emitting a spherical wave and, at any position, the new wavefront is determined by the sum of these secondary waves [14]. Given this principle, the complex amplitude of the electric field reflected by the IRS, denoted by $E_{r}\left(\mathbf{r}_{p}\right)$, at an arbitrary observation point $\mathbf{r}_{p}=\left(x_{p}, y_{p}, z_{p}\right)$, see Fig. 1], is given by [14]

$$
E_{r}\left(\mathbf{r}_{p}\right)=\frac{1}{j \lambda} \iint_{\Sigma} E_{i}(\mathbf{r}) \frac{\exp \left(j k\left|\mathbf{r}_{o p}\right|\right)}{\left|\mathbf{r}_{o p}\right|} \cos \left(\theta_{o p}\right) e^{j \Phi_{\mathrm{irs}}(x, y)} \mathrm{d} x \mathrm{~d} y,
$$

where $\mathbf{r}_{o p}$ is the vector between an arbitrary point on the IRS, denoted by vector $\mathbf{r}$, and any observation point $\mathbf{r}_{p}, \theta_{o p}$ denotes the angle between vector $\mathbf{r}_{o p}$ and the $z$-axis, $\Sigma$ is the IRS area, and $\Phi_{\text {irs }}(x, y)$ denotes the phase shift introduced by the IRS. In (7), the total surface of the IRS is divided into infinitesimal parts with area $\mathrm{d} x \mathrm{~d} y$, and the light wave scattered by each part is modeled as a secondary source emitting a spherical wave, modeled by $\frac{\exp \left(j k\left|\mathbf{r}_{o p}\right|\right)}{\left|\mathbf{r}_{o p}\right|}$. The complex amplitudes of the secondary sources are proportional to the incident electric field, $E_{i}(\mathbf{r})$, and an additional phase shift term, $e^{j \Phi_{\text {irs }}(x, y)}$, is introduced by the IRS. The phases of the spherical 
sources, $k\left|\mathbf{r}_{o p}\right|$, play an important role in our analysis, and for tractability, we approximate $\left|\mathbf{r}_{o p}\right|$. The length of vector $\mathbf{r}_{o p}$, see Fig. 1, is given by

$$
\left|\mathbf{r}_{o p}\right|=\left|\mathbf{r}_{p}-\mathbf{r}\right|=\left[\left(x-x_{p}\right)^{2}+\left(y-y_{p}\right)^{2}+z_{p}^{2}\right]^{1 / 2}
$$

Let $d_{p}=\left|\mathbf{r}_{p}\right|$, then we obtain

$$
\frac{\left|\mathbf{r}_{o p}\right|^{2}}{d_{p}^{2}}=1+\frac{x^{2}+y^{2}}{d_{p}^{2}}-2 \frac{x x_{p}+y y_{p}}{d_{p}^{2}} .
$$

Applying the Taylor series expansion [15] with $(1+x)^{1 / 2}=1+\frac{1}{2} x-\frac{1}{8} x^{2}+\cdots$, we obtain

$$
\left|\mathbf{r}_{o p}\right|=\underbrace{d_{p}-\frac{x x_{p}+y y_{p}}{d_{p}}}_{=\mathrm{t}_{1}}+\underbrace{\frac{x^{2}+y^{2}}{2 d_{p}}}_{=\mathrm{t}_{2}}+\underbrace{\frac{\left(x^{2}+y^{2}-2\left(x x_{p}+y y_{p}\right)\right)^{2}}{8 d_{p}^{3}}}_{=\mathrm{t}_{3}}+\cdots .
$$

For the far-field approximation, it is assumed that the rays reflected by the IRS surface are parallel to each other and the rays have only a linear phase shift w.r.t. each other [16]. This approximation is equivalent to assuming a linear phase shift for the phase of the secondary sources w.r.t. the $x$ - and $y$-directions. In other words, only $\mathrm{t}_{1}$ in 10 is taken into account, and $\mathrm{t}_{2}$ and all higher orders terms are neglected. For the far-field assumption to hold, the impact of $\mathrm{t}_{2}$ in the argument of the exponential term, $k\left|\mathbf{r}_{o p}\right|$, should be much smaller than one period of the complex exponential, and thus,

$$
k \frac{x^{2}+y^{2}}{2 d_{p}} \ll 2 \pi
$$

The range of the relevant values for $x$ and $y$ in (7) is bounded by the beam widths of the incident electric field $2 w_{x}$ and $2 w_{y}$ (where the power of the incident beam drops by $\frac{1}{e^{4}}$ compared to the peak value) and the size of the IRS $L_{x}$ and $L_{y}$, i.e., $x_{e}=\min \left(\frac{L_{x}}{2}, w_{x}\right) \geq|x|$ and $y_{e}=\min \left(\frac{L_{y}}{2}, w_{y}\right) \geq|y|$. For practical lens and IRS sizes, we have $a_{0}, L_{x}, L_{y} \ll d_{r}$, and hence, $d_{p} \approx d_{r}$ holds, see Fig. 11. Thus, substituting $x_{e}$ and $y_{e}$ for $x$ and $y$ in (11), respectively, and defining the far-field distance as follows

$$
d_{f}=\frac{x_{e}^{2}+y_{e}^{2}}{8 \lambda}
$$

for distances $d_{r} \approx d_{p} \gg d_{f}$, the approximation of (10) in (7) by only term $t_{1}$ is appropriate. However, depending on the values of $x_{e}$ and $y_{e}$, and the observation distance from the IRS, $d_{r}$, $d_{r} \gg d_{f}$ might not hold. For example, consider a typical IRS size of $L_{x}=L_{y}=50 \mathrm{~cm}$ and 


$$
\begin{aligned}
& E_{r}\left(\mathbf{r}_{r}\right)=C \frac{\pi \sin \theta_{r}}{4 \sqrt{b_{x} b_{y}}} e^{-\frac{k^{2}}{4 b_{x}}\left(X-\Phi_{x}\right)^{2}-\frac{k^{2}}{4 b_{y}}\left(Y-\Phi_{y}\right)^{2}} \\
& \times\left[\operatorname{erf}\left(\sqrt{b_{x}} \frac{L_{x}}{2}-\frac{j k}{2 \sqrt{b_{x}}}\left(X-\Phi_{x}\right)\right)-\operatorname{erf}\left(-\sqrt{b_{x}} \frac{L_{x}}{2}-\frac{j k}{2 \sqrt{b_{x}}}\left(X-\Phi_{x}\right)\right)\right] \\
& \times\left[\operatorname{erf}\left(\sqrt{b_{y}} \frac{L_{y}}{2}-\frac{j k}{2 \sqrt{b_{y}}}\left(Y-\Phi_{y}\right)\right)-\operatorname{erf}\left(-\sqrt{b_{y}} \frac{L_{y}}{2}-\frac{j k}{2 \sqrt{b_{y}}}\left(Y-\Phi_{y}\right)\right)\right]
\end{aligned}
$$

an FSO beam with wavelength of $\lambda=1550 \mathrm{~nm}$ illuminated from a LS at $\mathbf{p}_{i}=\left(d_{i}, \theta_{i}, \phi_{i}\right)=$ $\left(1000 \mathrm{~m}, \frac{\pi}{8}, 0\right)$. Then, the beam incident on the IRS has widths $w_{x}=0.52 \mathrm{~m}$ and $w_{y}=0.19 \mathrm{~m}$ and the far-field distance according to $(12)$ is $d_{f}=32.7 \mathrm{~km}$ and the observation point should be farther away from the center of the IRS than this distance, which is not practical. Thus, in order to obtain a model that is also valid for intermediate distances, we propose the following theorem which is valid for both intermediate and far-field distances. Assuming a linear phase shift profile across the IRS, a closed-form solution for the integral in (7) is determined in this theorem.

Theorem 1: Assuming a linear phase-shift profile across the IRS, i.e., $\Phi_{\text {irs }}(x, y)=k\left(\Phi_{x} x+\Phi_{y} y\right)$, where $\Phi_{x}$ and $\Phi_{y}$ are the constant phase-shift gradients in $x$ - and $y$-direction, respectively, then the electric field emitted by the LS at position $\mathbf{p}_{i}=\left(d_{i}, \theta_{i}, \phi_{i}\right)$ and reflected by the IRS at the lens located at $\mathbf{p}_{r}=\left(d_{r}, \theta_{r}, \phi_{r}\right)$ for any distance $d_{i} \gg L_{x}, L_{y}, d_{r} \gg a_{0}, L_{x}, L_{y}, d_{n}$, where

$$
d_{n}=\left[\frac{\left(x_{e}^{2}+y_{e}^{2}\right)^{2}}{8 \lambda}\right]^{1 / 3}
$$

is given by (14), shown on top of the next page. In $(14)$, we use $X=\varphi_{x}+c_{1} x_{r}+c_{2} y_{r}+2 \hat{b}_{x} x_{0}$, $Y=\varphi_{y}+c_{3} x_{r}+c_{4} y_{r}+2 \hat{b}_{y} y_{0}, C=\frac{E_{0} w_{0}}{j \lambda w\left(\tilde{d}_{i}\right) d_{r}} e^{j k\left(-\tilde{d}_{i}+d_{r}\right)+j k x_{0} \cos \left(\theta_{i}\right)+j \tan ^{-1}\left(\frac{\tilde{d}_{i}}{z_{0}}\right)-\nu \sin ^{2}\left(\theta_{i}\right) x_{0}^{2}-\nu y_{0}^{2}}, c_{1}=$ $\frac{1}{d_{r}} \cos \left(\phi_{r}\right) \sin \left(\theta_{r}\right), c_{2}=\frac{1}{d_{r}} \sin \left(\phi_{r}\right), c_{3}=\frac{-1}{d_{r}} \sin \left(\phi_{r}\right) \sin \left(\theta_{r}\right), c_{4}=\frac{1}{d_{r}} \cos \left(\phi_{r}\right), \varphi_{x}=\cos \left(\theta_{i}\right)+$ $\cos \left(\theta_{r}\right) \cos \left(\phi_{r}\right), \varphi_{y}=-\cos \left(\theta_{r}\right) \sin \left(\phi_{r}\right), b_{x}=\nu \sin ^{2}\left(\theta_{i}\right)-\frac{j k}{2 d_{r}}\left(1+\cos ^{2}\left(\phi_{r}\right) \cos ^{2}\left(\theta_{r}\right)\right), b_{y}=$ $\nu-\frac{j k}{2 d_{r}}\left(1-\sin ^{2}\left(\phi_{r}\right) \cos ^{2}\left(\theta_{r}\right)\right), \nu=\frac{1}{w^{2}\left(\tilde{d}_{i}\right)}+\frac{j k}{2 R\left(\tilde{d}_{i}\right)}, \hat{b}_{x}=-j \frac{b_{x}}{k}$, and $\hat{b}_{y}=-j \frac{b_{y}}{k}$.

Proof: The proof is given in Appendix B.

Eq. (14) explicitly shows the impact of the positioning of the LS and the lens w.r.t. the IRS, the size of the IRS, and the phase-shift configuration across the IRS on the electric field reflected by the IRS. In contrast to previous results for far-field approximations, the above theorem is 
valid even for intermediate distances. For the previous example, we obtain $d_{n}=9.4 \mathrm{~m}$ and for IRS-lens distances, $d_{r}$, larger than $d_{n}$, the result in $(14)$ is accurate. We may adopt the linear phase shifts proposed in previous works [3] to configure the IRS. In this case, $\Phi_{x}$ and $\Phi_{y}$ are set as follows [3]

$$
\begin{aligned}
& \Phi_{x}=\cos \left(\theta_{i}\right) \cos \left(\phi_{i}\right)+\cos \left(\theta_{r}\right) \cos \left(\phi_{r}\right), \\
& \Phi_{y}=\cos \left(\theta_{i}\right) \sin \left(\phi_{i}\right)+\cos \left(\theta_{r}\right) \sin \left(\phi_{r}\right) .
\end{aligned}
$$

In the following corollary, we consider a special case of Theorem 1 by assuming the conventional mirror. A conventional mirror introduces no additional phase shifts, i.e., $\Phi_{x}=\Phi_{y}=0$, and the incident angle and the reflection angle follow Snell's law, i.e., $\theta_{i}=\theta_{r}$.

Corollary 1 (Reflection by Conventional Mirror): Assume the size of the conventional mirror is very large, i.e., $L_{x}, L_{y} \gg 2 w\left(\tilde{d}_{i}\right)$, such that the entire received beam is reflected. Then, assuming a far-field scenario, $d_{r} \gg d_{f}, R\left(\tilde{d}_{i}\right) \rightarrow \infty, \phi_{r}=\pi-\phi_{i}$ and $x_{0}=y_{0}=0,14$ simplifies to

$$
E_{r}\left(\mathbf{r}_{r}\right)=C \pi w^{2}\left(\tilde{d}_{i}\right) \exp \left(-\frac{x_{r}^{2}+y_{r}^{2}}{w_{e q}^{2}}\right)
$$

where $w_{e q}=\frac{2 d_{r}}{k w\left(\tilde{d}_{i}\right)}$.

Proof: Considering $L_{x}, L_{y} \gg 2 w\left(\tilde{d}_{i}\right)$, we can substitute the $\operatorname{erf}(\cdot)$ terms in (14) by 4. Assuming $R\left(\tilde{d}_{i}\right) \rightarrow \infty$ and $d_{r} \gg d_{f}$, we obtain $b_{x}=\frac{\sin ^{2}\left(\theta_{i}\right)}{w^{2}\left(\tilde{d}_{i}\right)}$ and $b_{y}=\frac{1}{w^{2}\left(\tilde{d}_{i}\right)}$. Substituting $\theta_{i}=\theta_{r}, \phi_{r}=\pi$, and $\Phi_{x}=\Phi_{y}=0$, leads to 16 and this completes the proof.

Eq. (16) corresponds to a circular Gaussian beam, and reveals that in the assumed regime the reflected beam is identical to what is expected from geometrical optics under the far-field assumption, see [7].

Corollary 2 (Reflection by Anomalous Mirror): Consider an anomalous mirror which imposes the additional phase shifts, $\Phi_{x}$ and $\Phi_{y}$, given in $(15)$. Then, for the far-field scenario, $d_{r} \gg d_{f}$, $R\left(\tilde{d}_{i}\right) \rightarrow \infty, L_{x}, L_{y} \gg 2 w\left(\tilde{d}_{i}\right)$, and $x_{0}=y_{0}=0,14$ simplifies to

$$
E_{r}\left(\mathbf{r}_{r}\right)=\frac{C \pi\left|\sin \left(\theta_{r}\right)\right| w^{2}\left(d_{i}\right)}{\left|\sin \left(\theta_{i}\right)\right|} \exp \left(-\frac{k^{2} w^{2}\left(d_{i}\right)}{4 d_{r}^{2}}\left(\frac{\sin ^{2}\left(\theta_{r}\right) x_{r}^{2}}{\sin ^{2}\left(\theta_{i}\right)}+y_{r}^{2}\right)\right) .
$$

Proof: Substituting $\Phi_{x}$ and $\Phi_{y}$, given by (15), compensates for $\varphi_{x}$ and $\varphi_{y}$ in (14), respectively. Then, considering $\theta_{i} \neq \theta_{r}$ and following similar steps as in the proof of Corollary 1 leads to (17). This completes the proof.

Eq. (17) describes an elliptical Gaussian beam. This result is in agreement with the result from 
geometric optics for far-field reflection by an IRS [10].

Depending on the IRS size, distances $d_{r} \gg d_{f}$ might not be in the practical range for FSO systems, see Section IV. Therefore, Corollaries 1 and 2, which are in-line with the far-field approximation, do not always provide a valid result. Thus, Theorem 1 is required to determine the channel gain for practical applications, see Section IV.

\section{B. Deterministic Channel Gain of the IRS}

Given (14), the power distribution, $I_{\text {irs }}\left(\mathbf{r}_{r}\right)$, and the corresponding deterministic channel gain, $h_{\text {irs }}$, can be obtained with (4) and (3), respectively. In the following theorem, we assume $d_{r} \gg d_{n}$ for the distance of the lens from the IRS and provide the deterministic channel gain.

Theorem 2 (Out-of-Plane Reflection): Assume $d_{i} \gg L_{x}, L_{y}, d_{r} \gg a_{0}, L_{x}, L_{y}, d_{n}$, a linear phase-shift configuration of the IRS, $\Phi_{\text {irs }}=k\left(\Phi_{x} x+\Phi_{y} y\right)$, and a LS located at $\mathbf{p}_{i}$. Then, the channel gain for the lens at position $\mathbf{p}_{r}$ is given by

$$
\begin{aligned}
h_{\mathrm{irs}}^{\text {out }}=\frac{C_{h}}{2} \sqrt{\frac{\pi}{\rho_{x}}} \int_{-\varepsilon}^{\varepsilon} \exp \left(-\rho_{y} y_{r}^{2}-\varrho_{y} y_{r}+\frac{\left(\rho_{x y} y_{r}+\varrho_{x}\right)^{2}}{4 \rho_{x}}\right) \\
\times\left[\operatorname{erf}\left(\frac{2 \rho_{x}+\rho_{x y} y_{r}+\varrho_{x}}{2 \sqrt{\rho_{x}}}\right)-\operatorname{erf}\left(\frac{\rho_{x y} y_{r}-2 \rho_{x}+\varrho_{x}}{2 \sqrt{\rho_{x}}}\right)\right] \mathrm{d} y_{r},
\end{aligned}
$$

where $\varepsilon=\frac{\sqrt{\pi} a_{0}}{2}, \tilde{b}_{i}=\frac{2 b_{i} \bar{b}_{i}}{b_{i}+\bar{b}_{i}}, \bar{b}_{i}=\operatorname{conj}\left(b_{i}\right), i \in\{x, y\}, \rho_{1}=\varrho_{y}+\varepsilon \rho_{x y}, \rho_{2}=\varrho_{y}-\varepsilon \rho_{x y}, \rho_{x}=$ $\frac{k^{2}}{2}\left(\frac{c_{1}^{2}}{\tilde{b}_{x}}+\frac{c_{3}^{2}}{\tilde{b}_{y}}\right), \rho_{y}=\frac{k^{2}}{2}\left(\frac{c_{2}^{2}}{\tilde{b}_{x}}+\frac{c_{4}^{2}}{\tilde{b}_{y}}\right), \rho_{x y}=k^{2}\left(\frac{c_{1} c_{2}}{\tilde{b}_{x}}+\frac{c_{3} c_{4}}{\tilde{b}_{y}}\right), \varrho_{x}=k^{2}\left(\frac{c_{1}}{\tilde{b}_{x}}\left(\varphi_{x}-\Phi_{x}\right)+\frac{c_{3}}{\tilde{b}_{y}}\left(\varphi_{y}-\Phi_{y}\right)\right)$, $\varrho_{y}=k^{2}\left(-\frac{c_{2}}{\tilde{b}_{x}}\left(\varphi_{x}-\Phi_{x}\right)+\frac{c_{4}}{\tilde{b}_{y}}\left(\varphi_{y}-\Phi_{y}\right)\right), \varpi_{x}=-\sqrt{b_{x}} \frac{L_{x}}{2}-\frac{j k}{2 \sqrt{b_{x}}}\left(\varphi_{x}-\Phi_{x}+2 \hat{b}_{x} x_{0}+\left(c_{1}+c_{2}\right) a\right)$, $\varpi_{y}=-\sqrt{b_{y}} \frac{L_{y}}{2}-\frac{j k}{2 \sqrt{b_{y}}}\left(\varphi_{y}-\Phi_{y}+2 \hat{b}_{y} y_{0}+\left(c_{3}+c_{4}\right) a\right), C_{2}=\left(\operatorname{erf}\left(\sqrt{b_{x}} L_{x}+\varpi_{x}\right)-\operatorname{erf}\left(\varpi_{x}\right)\right) \times$ $\left(\operatorname{erf}\left(\sqrt{b_{y}} L_{y}+\varpi_{y}\right)-\operatorname{erf}\left(\varpi_{y}\right)\right), C_{h}=\frac{\pi^{2}\left|C_{2}\right|^{2} E_{0}^{2} w_{0}^{2} \sin ^{2}\left(\theta_{r}\right)}{32 \eta\left|b_{x}\right|\left|b_{y}\right| \lambda^{2} w^{2}\left(\tilde{d}_{i}\right) d_{r}^{2}} e^{-2 \nu\left(\sin ^{2}\left(\theta_{i}\right) x_{0}^{2}+y_{0}^{2}\right)} e^{-\frac{k^{2}}{2}\left(\frac{\left(\varphi_{x}-\Phi_{x}\right)^{2}}{\tilde{b}_{x}}+\frac{\left(\varphi_{y}-\Phi_{y}\right)^{2}}{\tilde{b}_{y}}\right)}$.

Proof: The proof is given in Appendix C.

Theorem 2 specifies the channel gain of an IRS-assisted FSO system when the normal vector of the LS plane and the normal vector of the lens plane may lie in different planes, which is referred to as "out-of-plane reflection" [17]. This is in contrast to Snell's law which states that the reflected and incident beams should be in the same plane with $\theta_{i}=\theta_{r}$. However, by adopting a linear phase shift at the IRS, the direction of the reflected beam can be out of the incident beam plane. Moreover, (18) specifies the dependence of the channel gain on the IRS size, $L_{x}$ and $L_{y}$, the phase gradients across the IRS surface, $\Phi_{x}$ and $\Phi_{y}$, the lens radius, $a_{0}$, and the lens and LS positions, $\mathbf{p}_{r}$ and $\mathbf{p}_{i}$, respectively. Furthermore, the position of the center of the beam 
footprint on the IRS surface, $x_{0}$ and $y_{0}$, or in other words the position where the Gaussian beam intersects the IRS surface, affects the channel gain. This is expected since when the beam is not accurately tracked, only a fraction of total power is received by the lens which degrades the channel gain.

In the following corollary, we simplify $(18)$, for the case where the normal vector of the LS plane and the normal vector of the lens plane lie in the same plane, which is referred to as “in-plane-reflection" [17].

Corollary 3 (In-Plane Reflection): For in-plane reflection, $\phi_{r}=\pi-\phi_{i}$, and the channel gain is given by

$$
\begin{aligned}
h_{\mathrm{irs}}^{\mathrm{in}} & =\frac{C_{h} \pi}{4 \sqrt{\rho_{x} \rho_{y}}} \exp \left(\frac{\varrho_{x}^{2}}{4 \rho_{x}}+\frac{\varrho_{y}^{2}}{4 \rho_{y}}\right) \\
& \times\left[\operatorname{erf}\left(\sqrt{\rho_{x}} \varepsilon+\frac{\varrho_{x}}{2 \sqrt{\rho_{x}}}\right)-\operatorname{erf}\left(-\sqrt{\rho_{x}} \varepsilon+\frac{\varrho_{x}}{2 \sqrt{\rho_{x}}}\right)\right] \\
& \times\left[\operatorname{erf}\left(\sqrt{\rho_{y}} \varepsilon+\frac{\varrho_{y}}{2 \sqrt{\rho_{y}}}\right)-\operatorname{erf}\left(-\sqrt{\rho_{y}} \varepsilon+\frac{\varrho_{y}}{2 \sqrt{\rho_{y}}}\right)\right] .
\end{aligned}
$$

Proof: Since $\phi_{i}=0$ and $\phi_{r}=\pi$, the parameters in Theorem 2 simplify to $\rho_{x}=-\frac{k^{2}}{2 d_{r}^{2} \tilde{b}_{x}} \sin ^{2}\left(\theta_{r}\right)$, $\rho_{y}=-\frac{k^{2}}{2 d_{r}^{2} \tilde{b}_{y}}, \rho_{x y}=0, \varrho_{x}=\frac{k^{2}}{d_{r} \hat{b}_{x}} \sin \left(\theta_{r}\right)\left(\varphi_{x}-\Phi_{x}\right), \varrho_{y}=0, \varphi_{y}=0$, and $\varphi_{x}=-\cos \left(\theta_{r}\right)+\cos \left(\theta_{i}\right)$. Substituting these values, the integral in (30) simplifies to two independent integrals, which can be solved by applying [15, Eq. (2.33-1)]. Then, we obtain (19) and this completes the proof.

\section{BER Performance Analysis}

Assuming OOK modulation, the average BER, denoted by $P_{e}$, over a Gamma-Gamma fading channel is given by [18]

$$
P_{e}=\sum_{\ell=0}^{\infty}\left(\xi_{\ell}(\alpha, \beta) \gamma^{-\frac{\ell+\beta}{2}}+\xi_{\ell}(\beta, \alpha) \gamma^{-\frac{\ell+\alpha}{2}}\right)
$$

where

$$
\begin{aligned}
& \xi_{\ell}(\alpha, \beta)= \\
& \quad \frac{\sqrt{\pi}(2 \sqrt{2} \alpha \beta)^{\ell+\beta} \Gamma\left(\frac{\ell+\beta+1}{2}\right)}{2 \sin [\pi(\alpha-\beta)] \Gamma(\alpha) \Gamma(\beta) \Gamma(\ell-\alpha+\beta+1)(\ell+\beta) \ell !} .
\end{aligned}
$$

Here, $\Gamma(\cdot)$ is the Gamma function and the signal-to-noise ratio term is denoted by $\gamma=\frac{\left(h_{p} h_{\mathrm{irs}}\right)^{2} \sigma_{s}^{2}}{\sigma_{n}^{2}}$. 

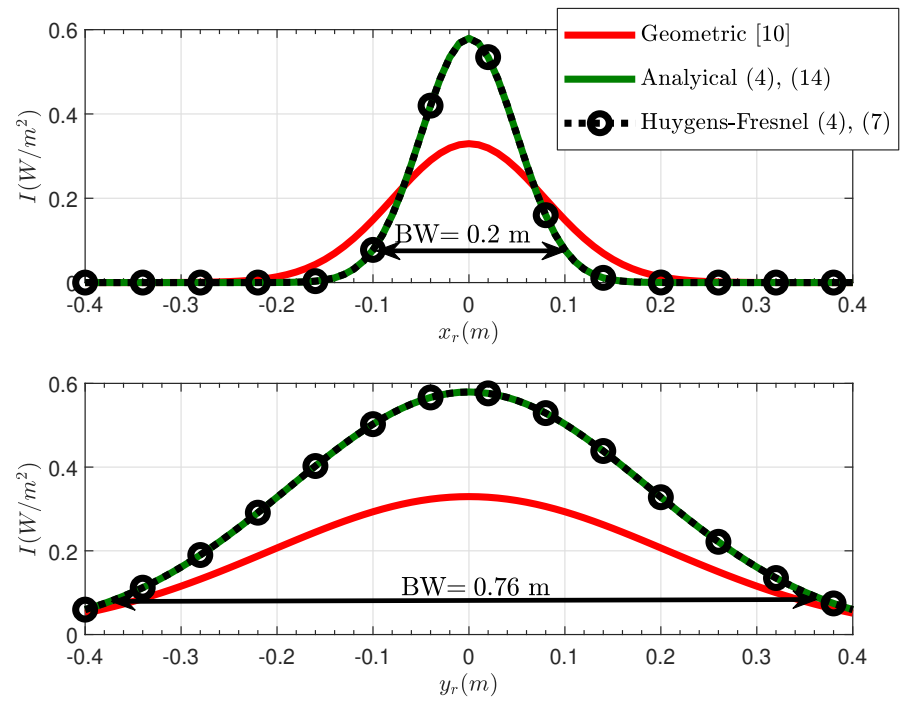

Fig. 2. Power density in the lens plane versus $x_{r}$ - and $y_{r}$.

\section{Simulation Results}

In this section, we validate our analytical results for the electric field in (14) and the channel gain of IRS-assisted FSO systems in (18) and investigate the BER performance. We consider a square-shaped IRS with $L_{x}=L_{y}=0.5 \mathrm{~m}$ and adopt the linear phase configuration given in 15$)$. The LS is positioned at $\mathbf{p}_{i}=\left(d_{i}, \theta_{i}, \phi_{i}\right)=\left(1000 \mathrm{~m}, \frac{\pi}{8}, 0\right)$ emitting a Gaussian beam with parameters $\lambda=1550 \mathrm{~nm}, E_{0}=1000 \frac{\mathrm{v}}{\mathrm{m}}$, and $w_{0}=2.5 \mathrm{~mm}$. The receiver lens is located at $\mathbf{p}_{r}=\left(d_{r}, \theta_{r}, \phi_{r}\right)=\left(2000 \mathrm{~m}, \frac{\pi}{2}, \pi\right)$. We adopt for the impedance of the propagation medium $\eta=377 \Omega$, for the Gamma-Gamma turbulence parameters $\alpha=2.1$ and $\beta=2$, and for the attenuation factor $\kappa=16.8 \times 10^{-3} \frac{\mathrm{dB}}{\mathrm{m}}$.

The top and bottom subfigures of Fig. 2 show the power density (4) of the Gaussian beam along the $x_{r}$ - and $y_{r}$-axes of the receiver lens coordinate system, respectively. Results for the power density according to our model in (14), numerical integration according to the HuygensFresnel principle using (7), which serves as ground truth, and geometric optics under the far-field approximation [10] are shown. Since for the beam width of the incident laser beam at the IRS, $w_{x}=0.52 \mathrm{~m} \simeq L_{x}$ and $w_{y}=0.19 \mathrm{~m} \leq L_{y}$ holds, the IRS can reflect most of the power it receives to the lens. As can be observed from Fig. 2, for the considered case, the result for the far-field approximation in [10] does not match the Huygens-Fresnel result. This is expected from our analysis since $d_{r}=2000 \mathrm{~m}$ is much smaller than the far-field distance, $d_{f}=32.7 \mathrm{~km}$, see (12). However, since the proposed model in (14) is valid for distances $d_{r} \gg d_{n}=9.4 \mathrm{~m}$, 


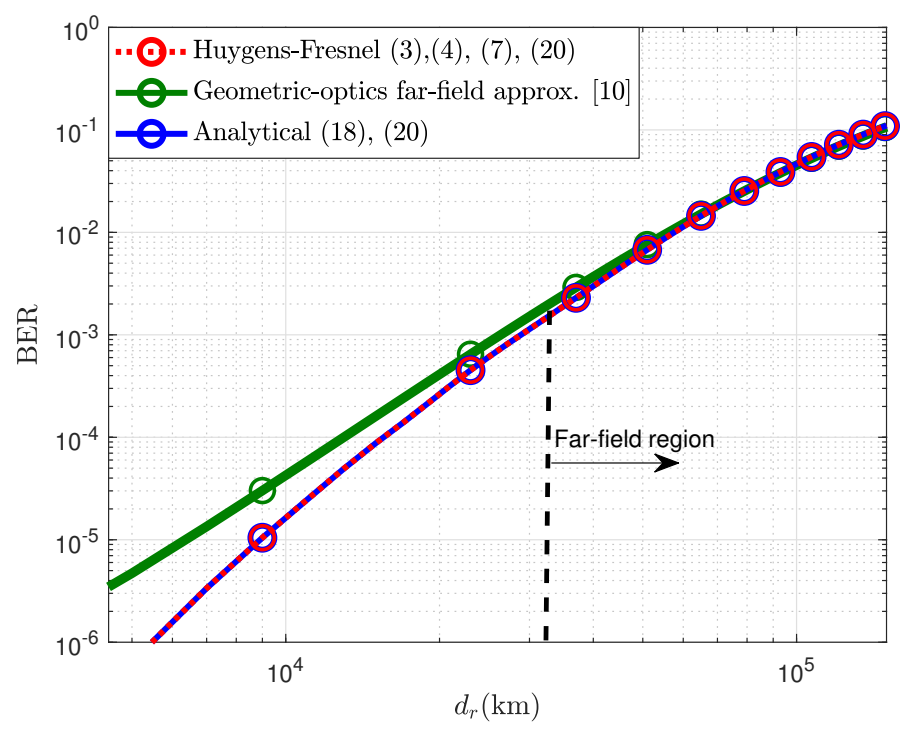

Fig. 3. BER performance versus $d_{r}$.

the power density obtained with this model perfectly matches the Huygens-Fresnel result. This confirms that, for practical IRS sizes and realistic IRS-lens distances, the proposed model has to be used to accurately model IRS-assisted FSO channels. Moreover, we observe in Fig. 2 that the beam on the lens plane is an elliptical Gaussian beam with beam widths $0.2 \mathrm{~m}$ and $0.76 \mathrm{~m}$ in the $x_{r}$ - and $y_{r}$ directions, respectively. While the IRS can adjust its phase-shift configuration such that the beam originating from the LS is reflected towards the lens, the circular shape of the laser beam changes to an ellipse on the lens.

Fig. 3 shows the BER performance as obtained with (20) for the considered setup versus the distance of the lens center, $d_{r}$, from the IRS. We validate the proposed model for the channel gain $h_{\text {irs }}$ (18) needed in 20 with numerical integration of the Huygens-Fresnel principle using (3), (4), and (7) and compare it with results for the geometric optics model with the far-field approximation in [10]. As can be observed, by increasing the IRS-lens distance, $d_{r}$, the BER performance degrades due to increased geometric and atmospheric losses. Moreover, the proposed model perfectly matches the results obtained with the Huygens-Fresnel principle for intermediate and far-field distances since for the considered $d_{r}, d_{r} \gg d_{n}=9.4 \mathrm{~m}$ holds. However, the BER for the far-field approximation approaches the Huygens-Fresnel result only for exceedingly large distances, i.e., for $d_{r}>d_{f}=32.7 \mathrm{~km}$. This confirms again that for practical applications of FSO systems, the far-field approximation does not yield realistic results and the proposed model has to be used instead. 


\section{Conclusions}

In this paper, we developed an analytical channel model for IRS-assisted FSO systems based on the Huygens-Fresnel principle. We determined the reflected electric field and the channel gain taking into account the non-uniform power distribution of Gaussian beams, the impact of the IRS size, the positions of the LS, the IRS, and the lens, and the phase-shift configuration of the IRS. We validated the accuracy of the proposed analytical model via simulations and showed that, in contrast to a model based on geometric optics employing the far-field approximation, the proposed model is valid even for intermediate distances, which are relevant in practice.

\section{APPENDiX A: Proof of LEMma 1}

First, we transform the LS plane coordinates $\mathbf{r}_{i}$ to Cartesian coordinates $\mathbf{r}$ as follows

$$
\mathbf{r}_{i}=\left(\mathbf{R}_{y_{i}}\left(\frac{\pi}{2}-\theta_{i}\right) \mathbf{R}_{z}\left(-\phi_{i}\right)\right)^{T}\left(\mathbf{r}-\mathbf{r}_{0}\right)+\left(0,0, d_{i}\right)
$$

where $\mathbf{R}_{z}(\phi)=\left(\begin{array}{ccc}\cos (\phi) & \sin (\phi) & 0 \\ -\sin (\phi) & \cos (\phi) & 0 \\ 0 & 0 & 1\end{array}\right)$ and $\mathbf{R}_{y}(\phi)=\left(\begin{array}{ccc}\cos (\phi) & 0 & -\sin (\phi) \\ 0 & 1 & 0 \\ \sin (\phi) & 0 & \cos (\phi)\end{array}\right)$ denote the counter-clockwise rotations by angle $\phi$ around the $z$ - and $y$-axes, respectively. Then, without loss of generality, we assumed $\phi_{i}=0$, thus, $\mathbf{R}_{\phi}=\mathbf{I}_{3}$. To project on the IRS plane, we substitute $z=0$ in 22, Then, assuming $d_{i} \gg L_{x}$, we can approximate $\hat{d}_{i} \approx \tilde{d}_{i}$ in the terms $w^{2}(\cdot), R(\cdot)$, and $\tan ^{-1}(\cdot)$ in (5). Then, by substituting $(22)$ in (5), we obtain $(6)$ and this completes the proof.

\section{APPEndix B: Proof of TheOREM 1}

First, we substitute $(6)$ into $(7)$ and use $e^{j \Phi_{\text {irs }}}=\exp \left(j k\left(\Phi_{x} x+\Phi_{y} y\right)\right)$ and $\cos \left(\theta_{o p}\right)=\frac{z_{p}}{\left|\mathbf{r}_{o p}\right|}$, which leads to

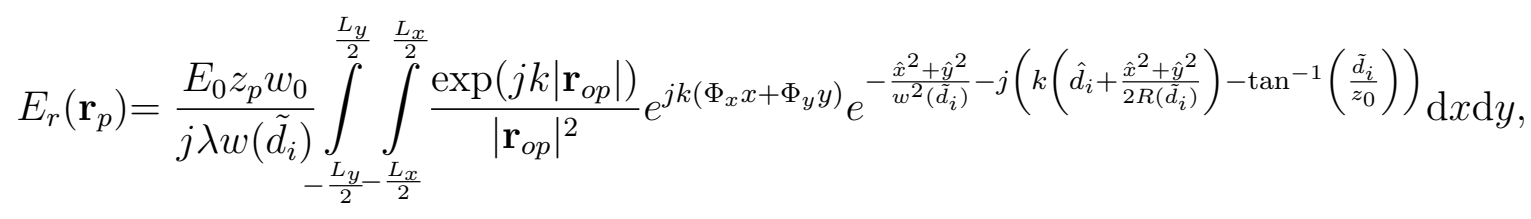

where we approximate $\left|\mathbf{r}_{o p}\right| \approx d_{p}+x^{2}\left(\frac{1}{2 d_{p}}+\frac{x_{p}^{2}}{2 d_{p}^{3}}\right)+y^{2}\left(\frac{1}{2 d_{p}}+\frac{y_{p}^{2}}{2 d_{p}^{3}}\right)-\frac{x x_{p}+y y_{p}}{d_{p}}$ by assuming in the exponential function $e^{j k\left|\mathbf{r}_{o p}\right|}$, the following term is sufficiently small,

$$
k \frac{\left(x^{2}+y^{2}\right)^{2}}{8 d_{p}^{3}} \ll 2 \pi .
$$


Substituting $x=x_{e}$ and $y=y_{e}$, we obtain

$$
\left(\frac{\left(x_{e}^{2}+y_{e}^{2}\right)^{2}}{8 \lambda}\right)^{1 / 3} \ll d_{p}
$$

and thus, for $d_{n}$ as defined in Theorem 11, our model is accurate for $d_{p} \gg d_{n}$. For the other terms that include $\left|\mathbf{r}_{o p}\right|$ in $(23)$, we approximate $\left|\mathbf{r}_{o p}\right| \approx d_{p}$ and thus, we substitute $\frac{z_{p}}{\left|\mathbf{r}_{o p}\right|^{2}}=\frac{z_{p}}{d_{p}^{2}}$. Now, we obtain from (23) that

$$
E_{r}\left(\mathbf{r}_{p}\right)=C_{1} \int_{-\frac{L_{x}}{2}}^{\frac{L_{x}}{2}} e^{-a x^{2}-b x} \mathrm{~d} x \int_{-\frac{L_{y}}{2}}^{\frac{L_{y}}{2}} e^{-c y^{2}-d y} \mathrm{~d} y
$$

where $C_{1}=\frac{E_{o} w_{0} z_{p}}{j \lambda w\left(\tilde{d}_{i}\right) d_{p}^{2}} e^{j k\left(-d_{i}+d_{p}\right)-\sin ^{2}\left(\theta_{i}\right) \nu x_{0}^{2}-\nu y_{0}^{2}+j k x_{0} \cos \left(\theta_{i}\right)+j \tan ^{-1}\left(\frac{\tilde{d}_{i}}{z_{0}}\right)}, a=\sin ^{2}\left(\theta_{i}\right) \nu-j k\left(\frac{1}{2 d_{p}}+\frac{x_{p}^{2}}{2 d_{p}^{3}}\right)$, $b=-2 x_{0} \sin ^{2}\left(\theta_{i}\right) \nu+j k\left(\cos \left(\theta_{i}\right)+\frac{x_{p}}{d_{p}}-\Phi_{x}\right), c=\nu-j k\left(\frac{1}{2 d_{p}}+\frac{y_{p}^{2}}{2 d_{p}^{3}}\right), d=-2 y_{0} \nu+j k\left(\frac{y_{p}}{d_{p}}-\Phi_{y}\right)^{p}$. Then, using [15, Eq. (2.33-1)] as follows

$$
\int e^{-a x^{2}-b x} \mathrm{~d} x=\frac{1}{2} \sqrt{\frac{\pi}{a}} \exp \left(\frac{b^{2}}{4 a}\right) \operatorname{erf}\left(\sqrt{a} x+\frac{b}{2 \sqrt{a}}\right),
$$

we can solve the integrals in 26. Next, we transform $\mathbf{r}_{p}$ from the $x y z$-coordinates to the lens plane coordinates. Thus, $\mathbf{r}_{r}=\mathbf{R}_{y_{r}}\left(\frac{\pi}{2}-\theta_{r}\right) \mathbf{R}_{z}\left(-\phi_{r}\right)\left(\mathbf{r}_{p}-\mathbf{r}_{0}\right)-\mathbf{r}_{r 0}$, where $\mathbf{r}_{r 0}=\left(0,0, d_{r}\right)$ is the translation vector to the reflection plane and $\mathbf{R}_{y}(\cdot)$ and $\mathbf{R}_{z}(\cdot)$ are rotation matrices defined in the proof of Lemma 1. Given the unitarity of matrices $\mathbf{R}_{y}(\cdot)$ and $\mathbf{R}_{z}(\cdot), \mathbf{r}_{p}$ can be expressed as $\mathbf{r}_{p}=\left(\mathbf{R}_{y_{r}}\left(\frac{\pi}{2}-\theta_{r}\right) \mathbf{R}_{z}\left(-\phi_{r}\right)\right)^{T}\left(\mathbf{r}_{r}+\mathbf{r}_{r 0}\right)+\mathbf{r}_{0}$, and thus, any point $\mathbf{r}_{p}$ on the lens plane is given by

$$
\mathbf{r}_{p}=\mathbf{r}_{0}+\left(\begin{array}{ccc}
\cos \left(\phi_{r}\right) \sin \left(\theta_{r}\right) & \sin \left(\phi_{r}\right) & \cos \left(\phi_{r}\right) \cos \left(\theta_{r}\right) \\
-\sin \left(\phi_{r}\right) \sin \left(\theta_{r}\right) & \cos \left(\phi_{r}\right) & -\sin \left(\phi_{r}\right) \cos \left(\theta_{r}\right) \\
-\cos \left(\theta_{r}\right) & 0 & \sin \left(\theta_{r}\right)
\end{array}\right)\left(\mathbf{r}_{r}+\mathbf{r}_{r 0}\right)
$$

Based on 28$)$ and since, $d_{r} \gg a, x_{0}, y_{0}$, we can approximate $d_{p} \approx d_{r}, z_{p} \approx d_{r} \sin \left(\theta_{r}\right), \frac{x_{p}^{2}}{d_{p}^{3}}=$ $\frac{1}{d_{r}} \cos ^{2}\left(\phi_{r}\right) \cos ^{2}\left(\theta_{r}\right)$, and $\frac{y_{p}^{2}}{d_{p}^{3}}=-\frac{1}{d_{r}} \sin ^{2}\left(\phi_{r}\right) \cos ^{2}\left(\theta_{r}\right)$. Finally, applying transformation 28 in (26) and (25), we obtain (14). This completes the proof. 


\section{Appendix C: Proof of Theorem 2}

First, we substitute (14) into (4) and since given the size of the lens, $\frac{x_{r}}{d_{r}}, \frac{y_{r}}{d_{r}} \ll 1$, we approximate $x_{r}=y_{r} \approx a_{0}$ in the $\operatorname{erf}(\cdot)$ terms in $(14)$ and substitute by $C_{2}$. After some simplification, we obtain

$$
\begin{aligned}
& h_{\mathrm{irs}}=\frac{\pi^{2}\left|C_{2}\right|^{2} E_{0}^{2} w_{0}^{2} \sin ^{2}\left(\theta_{r}\right)}{32 \eta\left|b_{x}\right|\left|b_{y}\right| \lambda^{2} w^{2}\left(\tilde{d}_{i}\right) d_{r}^{2}} e^{-2 \nu\left(\sin ^{2}\left(\theta_{i}\right) x_{0}^{2}+y_{0}^{2}\right)} \times \\
& \int_{-a_{0}}^{a_{0}} \int_{-\epsilon}^{\epsilon} \exp \left(\frac{-k^{2}}{2 \tilde{b}_{x}}\left(\bar{X}-\Phi_{x}\right)^{2}\right) \exp \left(\frac{-k^{2}}{2 \tilde{b}_{y}}\left(\bar{Y}-\Phi_{y}\right)^{2}\right) \mathrm{d} y_{r} \mathrm{~d} x_{r},
\end{aligned}
$$

where $\bar{X}=\varphi_{x}+c_{1} x_{r}+c_{2} y_{r}, \bar{Y}=\varphi_{y}+c_{3} x_{r}+c_{4} y_{r}$, and $\epsilon=\sqrt{a_{0}^{2}-x_{r}^{2}}$. Next, given the small size of the circular lens, we approximate it by a square with the same area and length $\sqrt{\pi} a_{0}$ and rewrite (29) as follows

$$
h_{\mathrm{irs}}=C_{h} \int_{-\varepsilon}^{\varepsilon} \int_{-\varepsilon}^{\varepsilon} e^{-\left(\rho_{x} x_{r}^{2}+\rho_{y} y_{r}^{2}+\rho_{x y} x_{r} y_{r}+\varrho_{x} x_{r}+\varrho_{y} y_{r}\right)} \mathrm{d} x_{r} \mathrm{~d} y_{r} .
$$

Then, we solve the inner integral by applying [15, Eq. (2.33-1)], which leads to (18) for out-ofplane reflection and completes the proof.

\section{REFERENCES}

[1] A. Arbabi, Y. Horie, M. Bagheri, and A. Faraon, "Dielectric metasurfaces for complete control of phase and polarization with subwavelength spatial resolution and high transmission," Nature nanotechnology, vol. 10, 082015.

[2] M. D. Renzo, A. Zappone, M. Debbah, M.-S. Alouini, C. Yuen, J. de Rosny, and S. Tretyakov, "Smart radio environments empowered by reconfigurable intelligent surfaces: How it works, state of research, and road ahead," ArXiv Preprints, April 2020. [Online]. Available: https://arxiv.org/abs/2004.09352

[3] M. Najafi, V. Jamali, R. Schober, and V. H. Poor, "Physics-based modeling and scalable optimization of large intelligent reflecting surfaces," ArXiv Preprints, 2020. [Online]. Available: https://arxiv.org/abs/2004.12957

[4] N. M. Estakhri and A. Alú, "Wave-front transformation with gradient metasurfaces," PHYSICAL REVIEW X, vol. 6, 2016. [Online]. Available: https://arxiv.org/abs/2004.09352

[5] E. Kochkina, G. Wanner, D. Schmelzer, M. Tröbs, and G. Heinzel, "Modeling of the general astigmatic Gaussian beam and its propagation through 3D optical systems," Appl. Opt., vol. 52, no. 24, pp. 6030-6040, Aug 2013.

[6] E. Basar, M. Di Renzo, J. De Rosny, M. Debbah, M. Alouini, and R. Zhang, "Wireless communications through reconfigurable intelligent surfaces," IEEE Access, vol. 7, 2019.

[7] M. Najafi and R. Schober, "Intelligent reflecting surfaces for free space optical communications," in Proc. IEEE Globecom, 2019.

[8] Z. Cao, X. Zhang, G. Osnabrugge, J. Li, I. Vellekoop, and A. Koonen, "Reconfigurable beam system for non-line-of-sight free-space optical communication," Light: Science and Applications, vol. 8, July 2019. 
[9] A. M. A. Abdelhady, A. S. Salem, O. Amin, B. Shihada, and M.-S. Alouini, "VLC via intelligent reflecting surfaces: Metasurfaces vs mirror arrays," 2020. [Online]. Available: http://hdl.handle.net/10754/661379

[10] M. Najafi, B. Schmauss, and R. Schober, "Intelligent reconfigurable reflecting surfaces for free space optical communications," ArXiv Preprints, May 2020. [Online]. Available: https://arxiv.org/abs/2005.04499

[11] M. Najafi, H. Ajam, V. Jamali, P. D. Diamantoulakis, G. K. Karagiannidis, and R. Schober, "Statistical modeling of FSO fronthaul channel for drone-based networks," in Proc. IEEE Int. Conf. Commun. (ICC), May 2018.

[12] M. Uysal, J. Li, and M. Yus, "Error rate performance analysis of coded free-space optical links over gamma-gamma atmospheric turbulence channels," IEEE Trans. Wireless Commun., vol. 5, no. 6, June 2006.

[13] B. E. A. Saleh and M. C. Teich, Fundamentals of Photonics. New York: Wiley, 1991.

[14] J. W. Goodman, Introduction to Fourier Optics. Roberts \& Co., 2005.

[15] I. S. Gradshteyn and I. M. Ryzhik, Table of Integrals, Series, and Products. San Diego, CA: Academic, 1994.

[16] E. Hecht, Optics. Pearson, 2017.

[17] F. Aieta, P. Genevet, N. Yu, M. A. Kats, Z. Gaburro, and F. Capasso, "Out-of-plane reflection and refraction of light by anisotropic optical antenna metasurfaces with phase discontinuities," Nano Letters, vol. 12, no. 3, pp. 1702-1706, 2012.

[18] E. Bayaki, R. Schober, and R. K. Mallik, "Performance analysis of MIMO free-space optical systems in gamma-gamma fading," IEEE Trans. on Commun., vol. 57, no. 11, pp. 3415-3424, 2009. 